\title{
Spatial Evolution of Filamentary Surface Discharges in High Magnetic Fields
}

\author{
P. Uhlig, J. C. Maan, and P. Wyder \\ Hochfeld-Magnetlabor, Max-Planck-Institut für Festkörperforschung, BP 166X, \\ F-38042 Grenoble CEDEX, France
}

(Received 15 August 1989)

\begin{abstract}
We present experiments on the effect of a transverse magnetic field ( 0 to $12 \mathrm{~T}$ ) on surface discharges (Lichtenberg figures) in different dense gases $\left(\mathrm{N}_{2}\right.$, air, $\mathrm{CO}_{2}$, and $\left.\mathrm{SF}_{6}\right)$. The formation of circular-shaped current filaments can be explained by a simple model. The particular dependence of the radius of curvature on the magnetic field observed $\left(R \propto B^{-1.5}\right)$ is thought to be due to local Hall fields. The structure of the discharge pattern can be tuned gradually with increasing magnetic field from a nonbranched, open morphology towards a dense, highly branched structure.
\end{abstract}

PACS numbers: $52.20 . \mathrm{Dq}, 52.80 . \mathrm{Mg}$

Filamentary gas discharges on insulating surfaces exhibit remarkable similarities ${ }^{1}$ to breakdown phenomena in long air gaps, ${ }^{2}$ e.g., to atmospheric lightning, and thus offer the possibility to perform well-defined "model" experiments in the laboratory. The application of sophisticated diagnostic tools, such as streak cameras, highspeed oscilloscopes, and time-resolved spectroscopy, ${ }^{3}$ in surface discharge experiments has improved the basic understanding of how a highly conducting phase-the filamentary "leader" channel-advances into a nonconducting medium - the surrounding gas. The interpretation of the spatiotemporal evolution of the discharge needs accurate experimental data on the dynamics of the fast-moving ionization region at the leader channel tips. Because of the nanosecond time scale involved and the inherent stochasticity of the discharge evolution, local measurements on the ionization region are difficult and can only be performed by guiding the discharge artificially (linear discharges). ${ }^{1,3,4}$ Morphological studies are no longer possible, an aspect which attracts significant interest since Niemeyer, Pietronero, and Wiesmann ${ }^{5}$ have shown that the branched discharge patterns have self-similar properties, i.e., are physical realizations of a "fractal."

In our experiment we apply a transverse high magnetic field during the discharge evolution and thus we avoid any spatial restriction of the surface discharge in order to use a locally sensitive probe. Since the Lorentz force acts on the fast-moving electrons, it is particularly effective in the high-field regions at the leader tips, where the channel formation takes place. In this sense, the magnetic field interferes with the dynamics of the early buildup phase of breakdown. With the magnetic field, the cyclotron radius is introduced as a new tunable length scale, on which the structural development of the discharge can be probed.

When a rectangular high-voltage pulse of about 500 ns length, $20 \mathrm{kV}$ amplitude, and less than $10 \mathrm{~ns}$ risetime is applied to a point-to-plane electrode system (Fig. 1), the electrodes being separated by a thin dielectric film (thickness $\simeq 100 \mu \mathrm{m}$ ), a discharge propagates in the gas just above the surface of the film. The discharge pattern is recorded directly with high resolution by using a photographic film as dielectric plate (Lichtenberg figure ${ }^{6}$ technique). The discharge chamber, which can be evacuated and refilled with pure gases $\left(\mathrm{N}_{2}, \mathrm{CO}_{2}\right.$, and $\left.\mathrm{SF}_{6}\right)$, is mounted into a Bitter magnet, supplying a homogeneous magnetic field up to $12 \mathrm{~T}$ in a $160-\mathrm{mm}$ bore. The field is oriented perpendicular to the dielectric plate.

Figure 2 shows the spatial evolution of a negative surface discharge in a nitrogen atmosphere as a function of the magnetic field. At $B=0$ a very bright starlike pattern develops. At moderate magnetic fields [up to $7 \mathrm{~T}$; Fig. 2(b)], the leader channels are bent and appear to have a circular shape outside the central electrode region. The radius of curvature is of the order of $1 \mathrm{~cm}$ at 7 T. The direction of the bending corresponds to the movement of electrons in crossed electric and magnetic fields. With increasing magnetic field the radius of curvature decreases, the channels approach each other and branching sets in. At the highest applied field of $12 \mathrm{~T}$ [Fig. 2(c)], circular-shaped current filaments are only found in the outmost regions of the discharge pattern where they can develop undisturbed by the fields of neighboring leader channels.

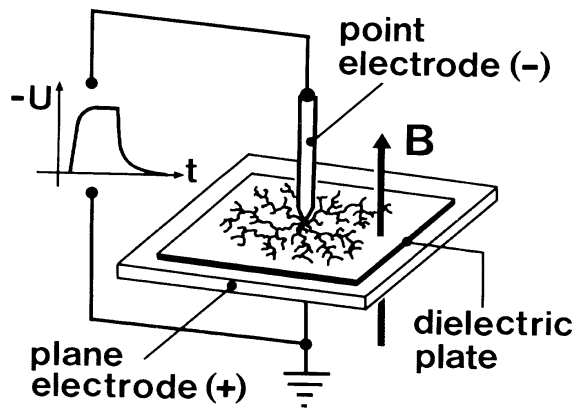

FIG. 1. Scheme of experimental setup to generate Lichtenberg figures in high magnetic fields. 


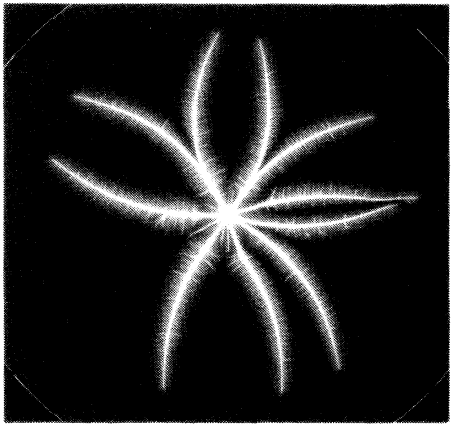

(a)

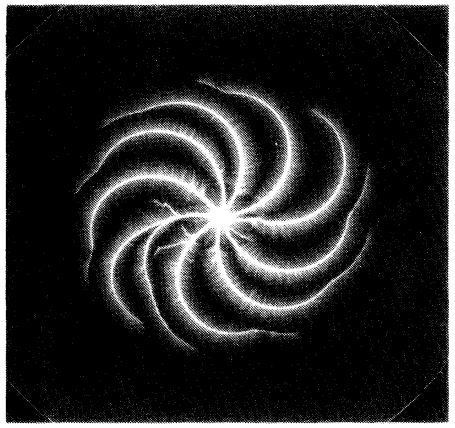

(b)

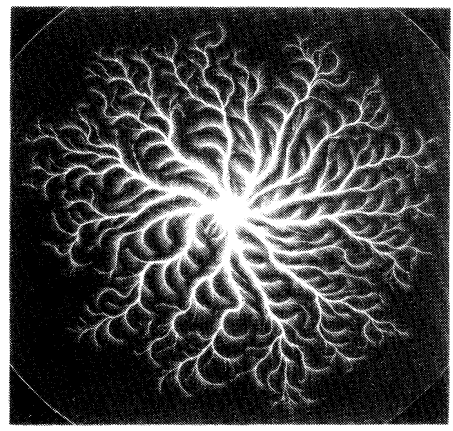

(c) $\rightarrow 1 \mathrm{~cm}$

FIG. 2. Surface discharge patterns in $\mathrm{N}_{2}$ at atmospheric pressure for different external magnetic fields, (a) $B=0 \mathrm{~T}$, (b) $B=5 \mathrm{~T}$, (c) $B=12 \mathrm{~T}$, for a negative point electrode $\left(V_{\text {appl }}=15 \mathrm{kV} ; 500 \mathrm{~ns}\right)$ on $100-\mu \mathrm{m}$ Mylar foil.

Figure 3 shows the field dependence of the observed bending of the current paths. The average radius of curvature follows a power law, $R \propto B^{-\alpha}$. We determine the exponent $\alpha$ for gases of different nature: $\alpha=1.53 \pm 0.17$ for the electropositive gas nitrogen, $\alpha=1.58 \pm 0.06$ for air, and $\alpha=1.27 \pm 0.15$ for the electronegative gas $\mathrm{CO}_{2}$. In all cases the discharge patterns are similar, leader branches are brighter for the more electronegative gases, and the absolute value of the radius is larger for $\mathbf{N}_{2}$. For the strongly electronegative gas $\mathrm{SF}_{6}$ we could not even observe parts of circular filaments. This seems to be a consequence of the pronounced noncontinuous, stepwise leader propagation ${ }^{7}$ in this gas.

From these results we draw the following conclusions: (1) The observed bending effect cannot be related to the movement of single charged particles in crossed electric and magnetic fields, which should result in curvatures of the order of $15 \mu \mathrm{m}$ at $1 \mathrm{~T}$ for electrons, i.e., the Larmor radius $r_{c}=v_{e} / \omega_{c}$ of the cyclotron orbit of an electron with enough kinetic energy to ionize gas molecules (ion-

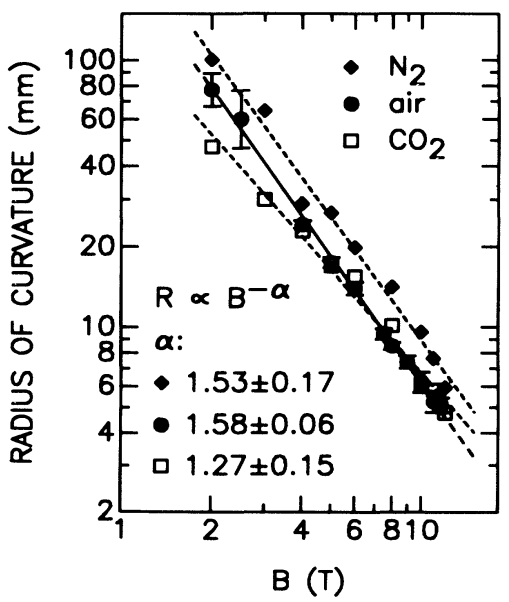

FIG. 3. Radius of curvature averaged over all individual filaments for applied magnetic field from 2 to $12 \mathrm{~T}$ in different gases at atmospheric pressure. ization energy $=16 \mathrm{eV}$ ). Because of the elevated collision frequency $v_{e 0}$ for electrons in a dense gas, ${ }^{8}$ an electron performs only a small part of a complete orbit $\left(\omega_{c} / v_{e} 0=0.04\right.$ at $\left.1 \mathrm{~T}\right)$; therefore, it has to be assumed that the macroscopic value of the radius of curvature results from the drift movement of the Larmor centers of the gyrating electrons. ${ }^{9}$ This bears a similarity to the current paths in metals or semiconductors in crossed $\mathbf{E}$ and $B$ fields, which are tilted against the driving electric field by the Hall angle $\theta_{H}$, where $\tan \theta_{H}=\omega_{c} \tau=\mu_{e} B$. (2) The circular shape of the current filaments is not expected for our point-to-plane electrode geometry: At the moment of application of the high-voltage pulse to the point electrode, the driving field has a radial symmetry. Since the electron drift direction is always tilted by the Hall angle with respect to the electric field, the current paths should be logarithmic spirals, a well-known result from magnetoresistance studies in solids in the so-called Corbino geometry, ${ }^{10}$ the analog of our "effective" electrode setup. The nonappearance of spiral-shaped filaments bears evidence that the ionizing electrons, which are responsible for the channel formation, are actually moving in the local fields established by the conducting leader channels themselves.

It is usually assumed that the transition between predischarge and leader is caused by the sudden thermal detachment of electrons from negative ions in the vicinity of the leader tip due to the Joule heating of the gas in the predischarge region. ${ }^{2}$ For our experimental conditions, leader channel and predischarge evolve in a quasistationary manner. This can be concluded from the practically constant value of the radius of curvature along a filament [Fig. 2(b)] and from the regular, continuous pattern of the predischarge corona streamers, which surround the channel from the electrode to the tip. This feature implies that the distribution of the charge densities of ions and electrons in the predischarge is stationary in a reference frame which is fixed to the advancing leader tip. At $B=0$, the distribution is symmetric to the axis of the leader channel and the transition from 


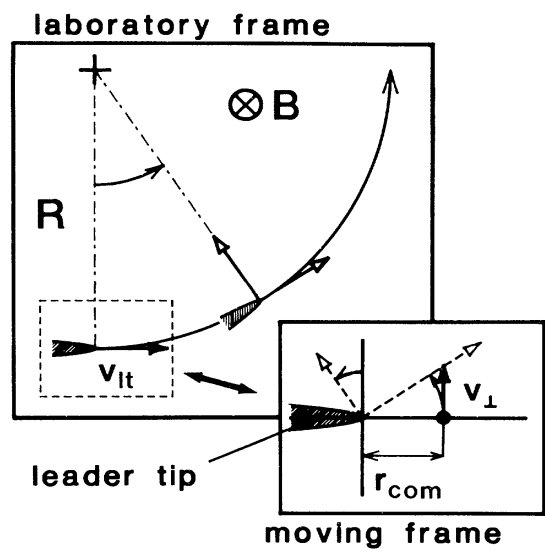

FIG. 4. Illustration of leader-tip propagation in a magnetic field in the laboratory frame resulting from the center-of-mass movement in a moving frame fixed to the tip.

predischarge to leader occurs on the axis in the vicinity of the tip. ${ }^{2,11}$ In a magnetic field the current flow is no longer parallel to the local electric field. The necessary rearrangement of the charges in order to reestablish quasistationary conditions could be obtained from a full two-dimensional solution of the continuity equations for charge, momentum, and energy conservation for all particle species involved and Poisson's equation to calculate the local electric fields. ${ }^{2,11-13}$ However, the essential features of discharge evolution in a magnetic field can be understood from a simplified approach. In a first approximation, we neglect the local electric fields due to the different displacements of the highly mobile electrons compared to the ions ("Hall fields"). In order to describe the reaction of the ensemble of electrons when switching on a magnetic field, we replace the whole distribution of electrons by a "representative" electron at the center-of-mass position $r_{\text {com }}$ in the moving reference frame (Fig. 4). Under the action of the Lorentz force the electron gets a perpendicular drift velocity $v_{\perp}$ $=v_{T} \omega_{c} \tau$, where $\mathbf{v}_{T}$ is the velocity parallel to $\mathbf{E}$ (note that $v_{T} \equiv v_{\text {lt }}$, where $v_{\text {lt }}$ is the velocity of the leader tip). To reestablish quasistationary conditions, the tip tends to follow the center-of-mass movement; this can be expressed by a rotation of the coordinate system fixed to the tip with the angular velocity $\Omega=v_{\perp} / r_{\text {com. }}$. In the laboratory frame the tip evolves along a circular path with a radius $R$ given by $\Omega=v_{\mathrm{lt}} / R \equiv v_{\perp} / r_{\text {com }}$. We obtain

$$
R=r_{\mathrm{com}} / \omega_{c} \tau=r_{\mathrm{com}} / \mu_{e} B \text {. }
$$

In the simplest approximation, $r_{\text {com }}$ may be estimated by the distance where the drift velocity of an electron in the Laplace field of the leader tip equals the tip velocity. Assuming an ideally conducting leader with parabolic profile, a tip radius of $25 \mu \mathrm{m}$, and an estimated tip potential of $\simeq 7 \mathrm{kV}$, we find $r_{\text {com }} \simeq 300 \mu \mathrm{m}$, and therefore $R=1 \mathrm{~mm}$ at $B=7 \mathrm{~T}$, still slightly smaller than the mea-

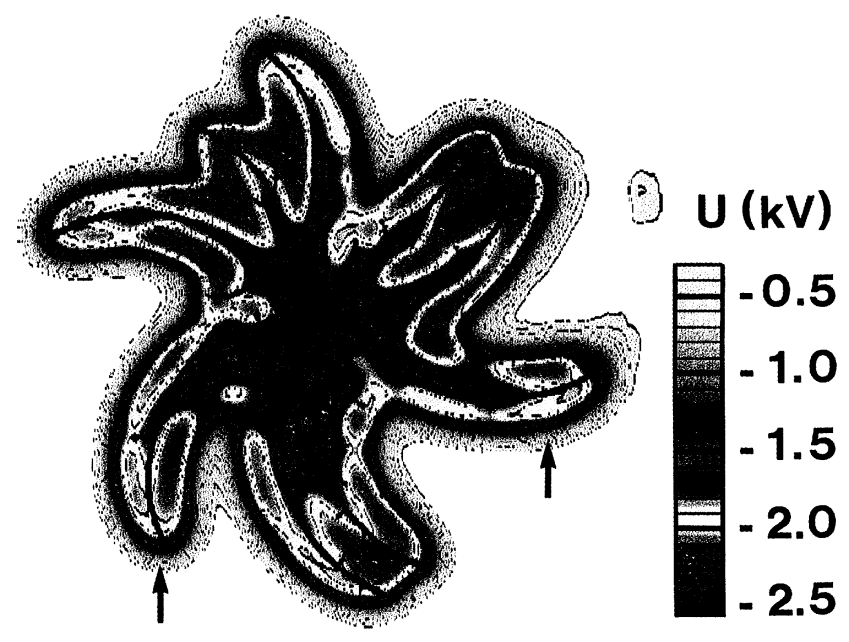

FIG. 5. Potential distribution of the charge left on a 100$\mu \mathrm{m}$ PETP support after a surface discharge in air at $B=4 \mathrm{~T}$, superimposed with the leader paths. Note the asymmetry in the distribution (arrows).

sured radius of curvature. As a consequence of quasistationary propagation, the radius of curvature is independent of the leader-tip velocity. From (1) it follows that $R$ should depend on the magnetic field as $B^{-1}$ and not as $B=-1.5$, as observed. To improve the model, we have to take into account the space-charge fields of the distributions of ions and electrons in the predischarge region. Because of their different mobilities, a transverse polarization-a Hall field-is created. As a consequence, the electric field direction at the center-of-mass position with respect to the channel axis will depend on the magnetic field strength; therefore, a stronger field dependence of the radius of curvature $R \propto B^{-(1+x)}$ $(x>0)$ can be expected. A detailed analysis of this aspect has not yet been done. On the other hand, quantitative measurements on the charge patterns induced by the surface discharge in thin Mylar [poly(ethylene terephthalate) (PETP)] foils have confirmed the existence of a transverse polarization in the predischarge in a magnetic field (Fig. 5). ${ }^{14}$

The complexity of the discharge pattern can be controlled by the magnetic field strength (Fig. 2). It appears that an increase in complexity is induced on the scale of the bending observed, and not on the microscopic scale of the cyclotron radius. The bending effect leads to an enhancement of screening of the local electric fields at the channel tips and at the concave side of the filaments, thus forcing the appearance of new branches at the convex side of the channels. A detailed analysis of the topological properties of surface discharges under the action of a magnetic field with regard to possible changes of the fractal dimension is in preparation. ${ }^{15}$

In summary, our experiments on filamentary surface discharges in high magnetic fields have shown that the 
complexity of the discharge pattern is controlled by the magnetic field strength. The bending effect on the filaments provides clear evidence that the distribution of charges in the predischarge region, determining the local conductivity, is crucial for the leader propagation. A simple model explains the occurrence of the circular shape of the filaments and the macroscopic order of the radius of curvature. The unusual magnetic field dependence of the latter demands for a refined model, including the effect of space-charge fields.

It is a pleasure to thank Dr. I. D. Vagner for many illuminating discussions.

${ }^{1}$ M. Töpler, Ann. Phys. (N.Y.) 21, 193 (1906).

${ }^{2}$ I. Gallimberti, J. Phys. (Paris), Colloq. 40, C7-197 (1979).

${ }^{3}$ S. Larigaldie, J. Appl. Phys. 61, 90 (1987).

${ }^{4} \mathrm{~L}$. Niemeyer and F. Pinnekamp, in Gaseous Dielectrics III, edited by E. Christopherou (Pergamon, New York, 1982), p. 379.
${ }^{5}$ L. Niemeyer, L. Pietronero, and H. J. Wiesmann, Phys. Rev. Lett. 52, 1033 (1984); L. Pietronero, A. Erzan, and C. Evertsz, Phys. Rev. Lett. 61, 861 (1988).

${ }^{6}$ G. C. Lichtenberg, Novi Commentarii Societatis Regiae Scientiarium Gottingae 8, 168 (1777).

${ }^{7}$ I. Gallimberti and N. Wiegart, J. Phys. D 12, 2351 (1986).

${ }^{8}$ A. J. Davies, C. S. Davies, and C. J. Evans, Proc. IEEE 118, 816 (1971).

${ }^{9}$ W. P. Allis, in Handbuch der Physik, edited by S. Flügge (Springer-Verlag, Berlin, 1956), Vol. XXI, p. 383.

${ }^{10}$ O. M. Corbino, Phys. Z. XII, 561 (1911).

${ }^{11}$ M. M. Kekez and P. P. Savic, in Electrical Breakdown and Discharges in Gases, edited by E. E. Kunhardt and L. H. Luessen (Plenum, New York, 1983), Vol. 89a, p. 419.

${ }^{12} \mathrm{E}$. Marode, in Electrical Breakdown and Discharges in Gases, edited by E. E. Kunhardt and L. H. Luessen (Plenum, New York, 1983), Vol. 89b, p. 119.

${ }^{13}$ I. Abbas and P. Bayle, J. Phys. D 14, 649 (1981).

${ }^{14}$ P. Uhlig, H. Beuter, B. Wanner, J. C. Maan, and P. Wyder (to be published).

${ }^{15}$ P. Uhlig, P. A. Darbon, J. C. Maan, and P. Wyder (to be published). 


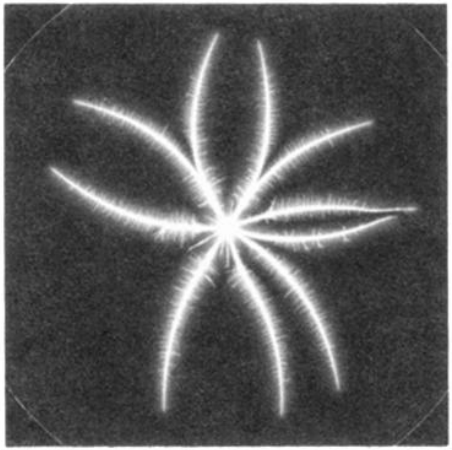

(a)

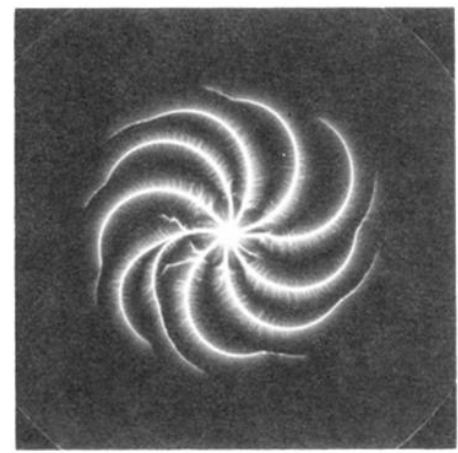

(b)

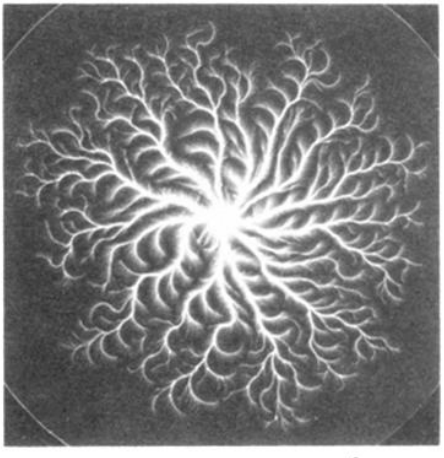

(c) $\rightarrow 1 \mathrm{~cm}$

FIG. 2. Surface discharge patterns in $\mathrm{N}_{2}$ at atmospheric pressure for different external magnetic fields, (a) $B=0 \mathrm{~T}$, (b) $B=5 \mathrm{~T}$, (c) $B=12 \mathrm{~T}$, for a negative point electrode $\left(V_{\text {appl }}=15 \mathrm{kV} ; 500 \mathrm{~ns}\right)$ on $100-\mu \mathrm{m}$ Mylar foil. 


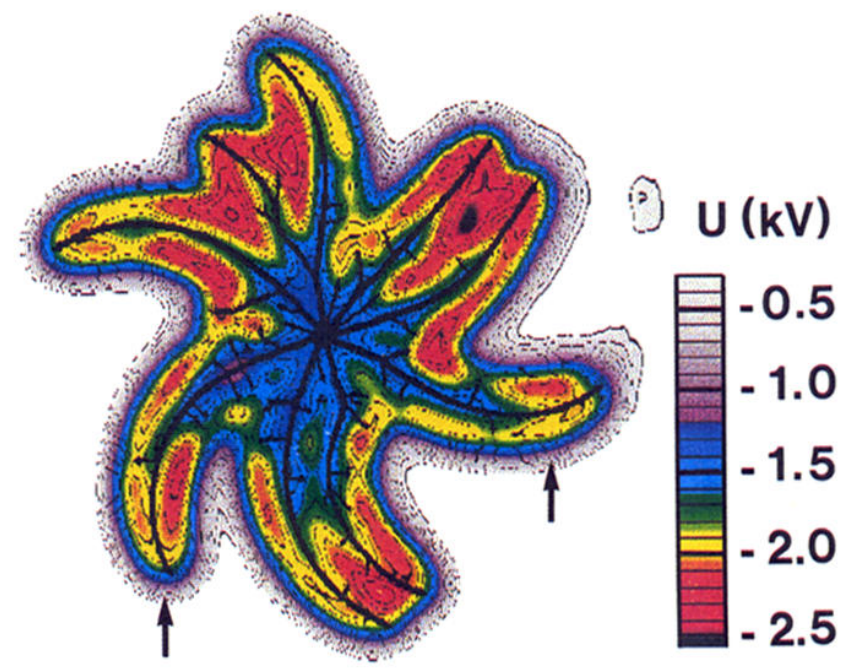

FIG. 5. Potential distribution of the charge left on a 100$\mu \mathrm{m}$ PETP support after a surface discharge in air at $B=4 \mathrm{~T}$, superimposed with the leader paths. Note the asymmetry in the distribution (arrows). 